E-ISSN 2347-2677

P-ISSN 2394-0522

www.faunajournal.com

IJFBS 2022; 9(1): 37-44

Received: 08-11-2021

Accepted: 23-12-2021

\section{CP Singh}

Department of Zoology, Wild life and Environmental Research

Lab, Janta Mahavidyalaya,

Ajitmal, Auraiya, Utter Pradesh

India

\section{Dr. Yogesh Babu Dixit}

Department of Zoology, Wild life and Environmental Research

Lab, Janta Mahavidyalaya,

Ajitmal, Auraiya, Utter Pradesh India

\section{Corresponding Author:}

CP Singh

Department of Zoology, Wild life

and Environmental Research

Lab, Janta Mahavidyalaya,

Ajitmal, Auraiya, Utter Pradesh

India

\section{Avian biodiversity in Bharthana and Chakar Nagar Tahseel of district Etawah U.P. India}

\author{
CP Singh and Dr. Yogesh Babu Dixit
}

DOI: https://doi.org/10.22271/23940522.2022.v9.i1a.878

\begin{abstract}
There were seven surveys conducted for the purpose of identification of avian diversity in Bharthana and Chakar Nager Tahseel of District Etawah in U.P. These ornithological surveys were carried out of three years from March 2017 to March 2021 for assessing the distribution, status, abundance of local, resident, migratory bird species in study area. Covering area is about $225 \mathrm{~km} 2$. In these surveys there were a total bird species 85 recorded and belonging to 72 genera, 37 families and over 15 orders were noticed, out of these 85 bird species diversity shows that $69.41 \%$ resident, $24.70 \%$ local migrant, $1.17 \%$ migrant, $4.70 \%$ summer visitors were distributed. No winter visitor species was observed. The habitat preference is wetland, terrestrial, terrestrial forest terrestrial aerial and terrestrial riverine. According to IUCN red list category shows that NT, VU, EN, LC and T were recorded in surrounding target area of study. The abundance $62.52 \%$ common, $28.25 \%$ un common and $8.25 \%$ rare bird species were found.
\end{abstract}

Keywords: Local water bodies, Resident birds, local migrant, winter visitors, summer visitors and IUCN

\section{Introduction}

Birds are flying vertebrates they comprises $13 \%$ of total birds species in Indian subcontinent in world the presence of more than 9000 Bird Species Girmett et al. $1988^{[1]}$. The habitat of birds is divided in to four categories that are forests, wetland, shrubs and riverine area but some species are found in both terrestrial and aquatic habitat, they have fantastic ability to move and most species are found in particular habitats. The living species of birds are grouped in to 27 Order and 155 families. Any environmental or biotic pressure or anthropogenic disturbance altering the habitat and distribution of plant community may seriously affected the bird's diversity. The species richness depend on availability of food, water bodies, plant community and less anthropogenic activities and absence of predators. These facilities makes easy and safe in feeding, moving, breeding and in parental caring. According to food birds are omnivorous, frugivorous, insectivorous, grain eater, carnivorous, sap feeder and herbivorous. The richness of species is mainly due to presence of adequate food supply, water quality and plant community and without any biotic disturbances. Bharthna tahseel (26.7523 N 79.2218 E) is located near NH2 in south direction from $8 \mathrm{Km}$ away from Bakewar kaswa (26.6617N,79.1746E), and $37 \mathrm{~km}$ away from district head quarter of Etawah district (26.7900N,79.0300E) and Chakar Nagar tahseel (26.5741 N,79.0956 E) is located in National Chambal Sanctuary area away from district head quarter about $36.7 \mathrm{~km}$. In the presence of a large number of water bodies and plant communities. Bharthana and Chakar Nager area is an ideal habitat of all kind of birds including local and migratory birds. Migratory birds visited in months of last November to April. The water bodies used to support water migratory aquatic as well as terrestrial bids population. There is no comprehensive data available till now for avian fauna in this area. So we decided to carry out a detailed study.

\section{Survey method and identification}

The entire study area was surveyed by motorcycle some time by car or on foot for observing birds population and habitat information. Data sheets were used to record all related aspects of avifauna such as habitat type, location, feeding habit, their number, activity and occurring time. Observations were carried out at regular intervals during the time of each months at weekly or sometimes fortnightly. The observations were made a period of three years from March 2017 to march 2021.A binocular (10x50) used for visual aids. Photographic evidences were taken by sonny camera (16 x mega pixel) or some times by Redmi Note 8 Mobile camera also used. Linear and branching surveys methods also have done ${ }^{[7]}$. 
Bird survey was conducted, when birds are most active during day from 07:00 to 11:00hrs and from 16:00 to 19:00 hrs. Field visits have been conducted weekly twice in the entire habitat. Identification manuals and field guides [8, 9, 17] were used during survey. A common, scientific name, Classification and Nomenclature of the birds following ${ }^{[10-13]}$ was adopted. The birds were categorized as Resident (R) and Migratory (M); Aquatic (A) and Terrestrial (T) ${ }^{[11]}$. All the birds species recorded during the present study were tabulated giving their scientific name, family, IUCN status \& W (P) A legal status. The following formula was used for calculating the percentage of familes orders and other aspects

$$
\text { Percentage of occurrence }=\frac{\text { No of each species of order/family }}{\text { Total no of different species }} \times 100
$$

\section{District Map of Etawah}

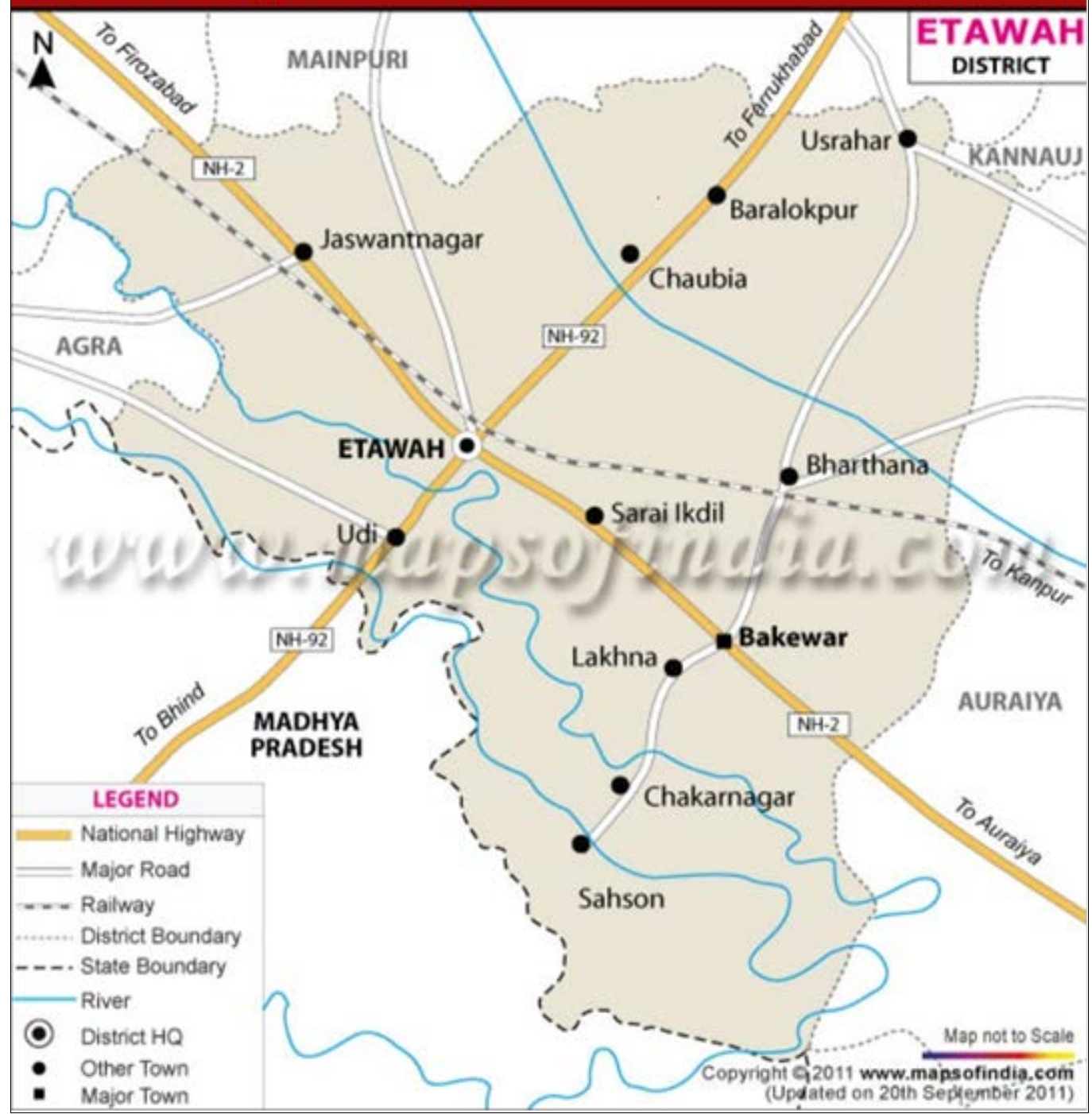

Fig 1: Map of Etawah

\section{Results and discussion}

Bharthana and Chakar nagar tahseel are located near north side of Chambal Sanctuary in District Etawah. The bird survey were conducted three consequent years from march 2017 to march 2021, a total 85 bird species belonging to 72 genera, 37 families and over 15 orders were recorded (Table 1 ). The percentage composition of families and genus under different orders are shown in Table 3 and Fig 1. Among the 15 orders of birds recorded, order Passeriformes is dominant with 11 families contributing $29.72 \%$ of the total species and 14 genus contributing $19.44 \%$ followed by Charadriiformes with 05 families (13.51\%), and 09 genus (12.5\%), Cuculiformes with 03 families (8.10\%) and 08 genus (11.11\%), Collumbiformes, Coracilformes Falconiformes Suliformes Bucerotiformes Pelecaniformes 02 families each
(5.40\%) and lowest families Galliformes, Gruiformes, Ciconiiformes, Apodiformes, Strigiformes, Psittaciformes each with 01 family (2.70\%) percentage of genus of Coracilformes and Cuculiformes 08 (11.11\%),07 (9.72\%) Falconiformes, 04 (5.55\%) Galliformes, Gruiformes Suliformes.03 (4.16\%) Ciconiiformes, Pelecaniformes, 02 (2.77\%) Collumbiformes Strigiformes, Bucerotiformes, lowest genus 01 (1.38\%) Psittaciformes Apodiformes, of the total 37 recorded families the percentage of the species are Ardeidae and Accipitridae contributed highest with 08(9.41\%) followed by Phasianidae and Clummbidae 05 (5.88\%), Cuculidae, Scolopacidae, Sturnidae, Ciconiidae 04 (4.70\%), 03(3.52\%), Alcedinidae, charadriidae, 02(2.35\%) Threskiornithidae Meropidae, Phalacrocoracidae corvidae Dicruridae, Strigidae, Leiothrichidae, Laridae, Ralidae, 
Gruidae, Coracidae, Pandionidae, Pelecanidae, Anhingidae, Burhinidae, Apodidae, Cisticolidae, Ploceidae, zosteropidae, Muscicapidae, Pycnonotidae, Motacillidae, Passeridae, Upupidae, Bucerotidae, Psittaculidae each with 01 (1.17\%). The habitat preference is $38.82 \%$ wet land, $30.58 \%$ terrestrial, $21.17 \%$ terrestrial forest. $4.70 \%$ terrestrial riverine and terrestrial aerial and $69.41 \%$ resident,24.70\% local migrant, $4.70 \%$ summer visitor, $1.17 \%$ migrant species were recorded.
The IUCN red list 2021 shows 77.64\% least concern, 8.23\% threatened, 5.88\% vulnerable and near threatened and $2.43 \%$ endangered. The abundance is $62.52 \%$ common, $28.25 \%$ un common and $8.25 \%$ rare species were observed. Main threats in the habitation are deforestation, use of chemical fertilizers pesticides insecticides and herbicides in agricultural fields, water lifting from water bodies poaching fishing were also noticed and polythene and sewage shown in table: 5 .

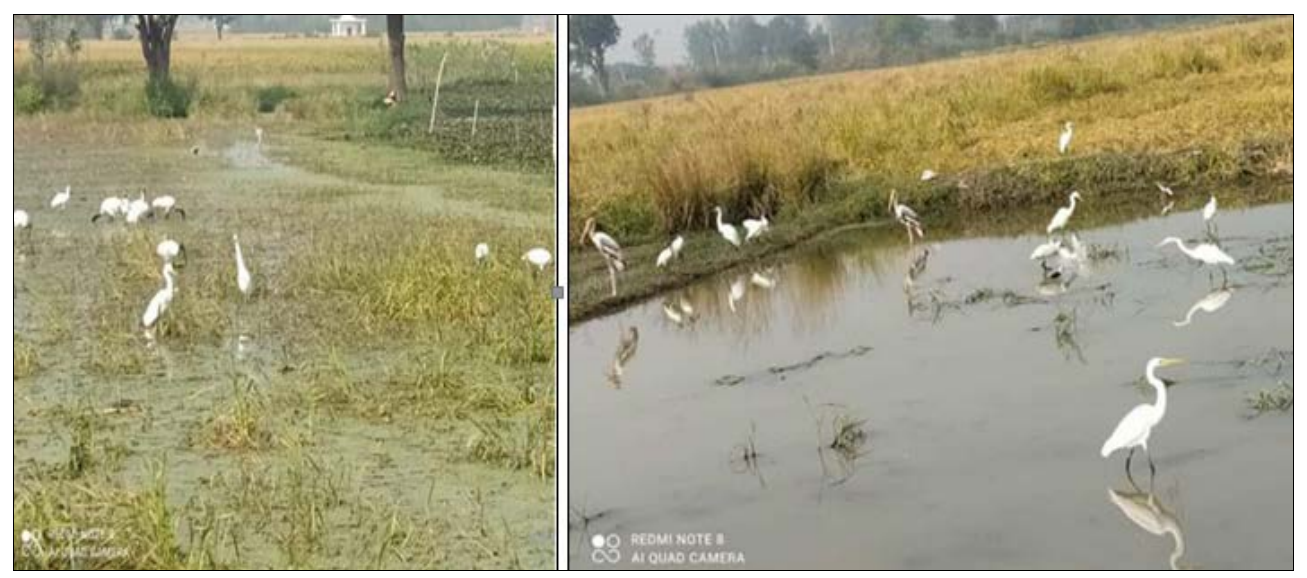

Fig 1, 2: Bird flock feeding in water body of Gadauna Phoolpur canal Bridge Auraiya

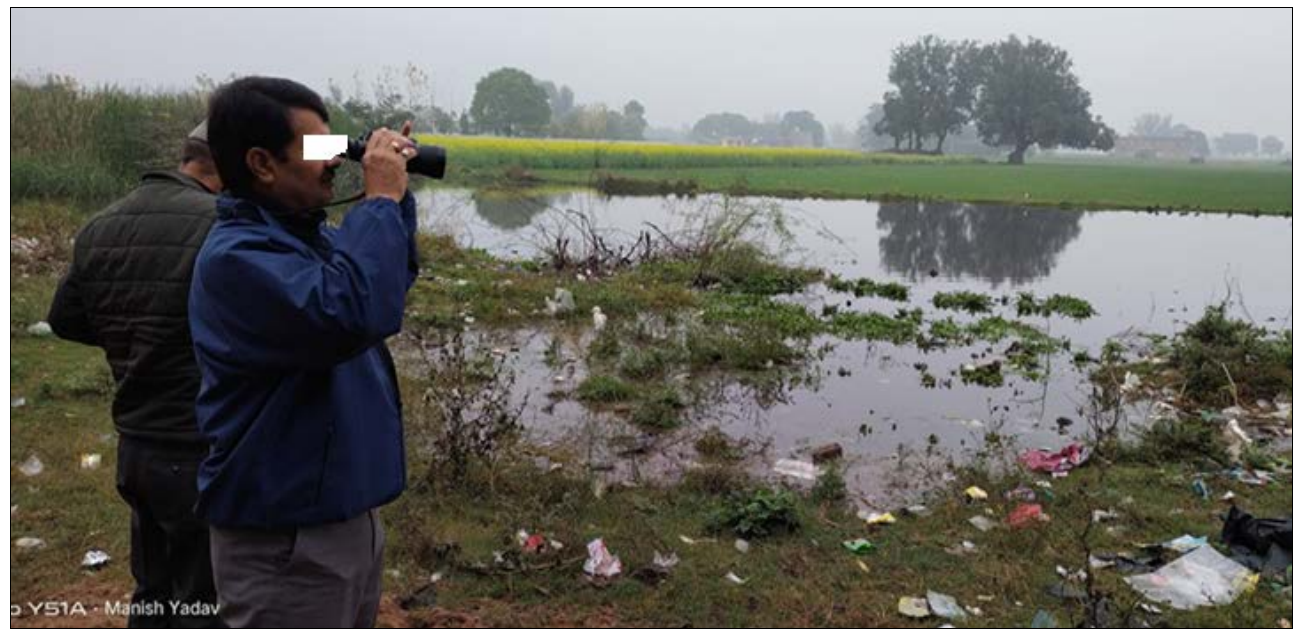

Fig 3: Dr Y. B, Dixit bird watching water body of Ajitmal Near J. M. V. Auraiya

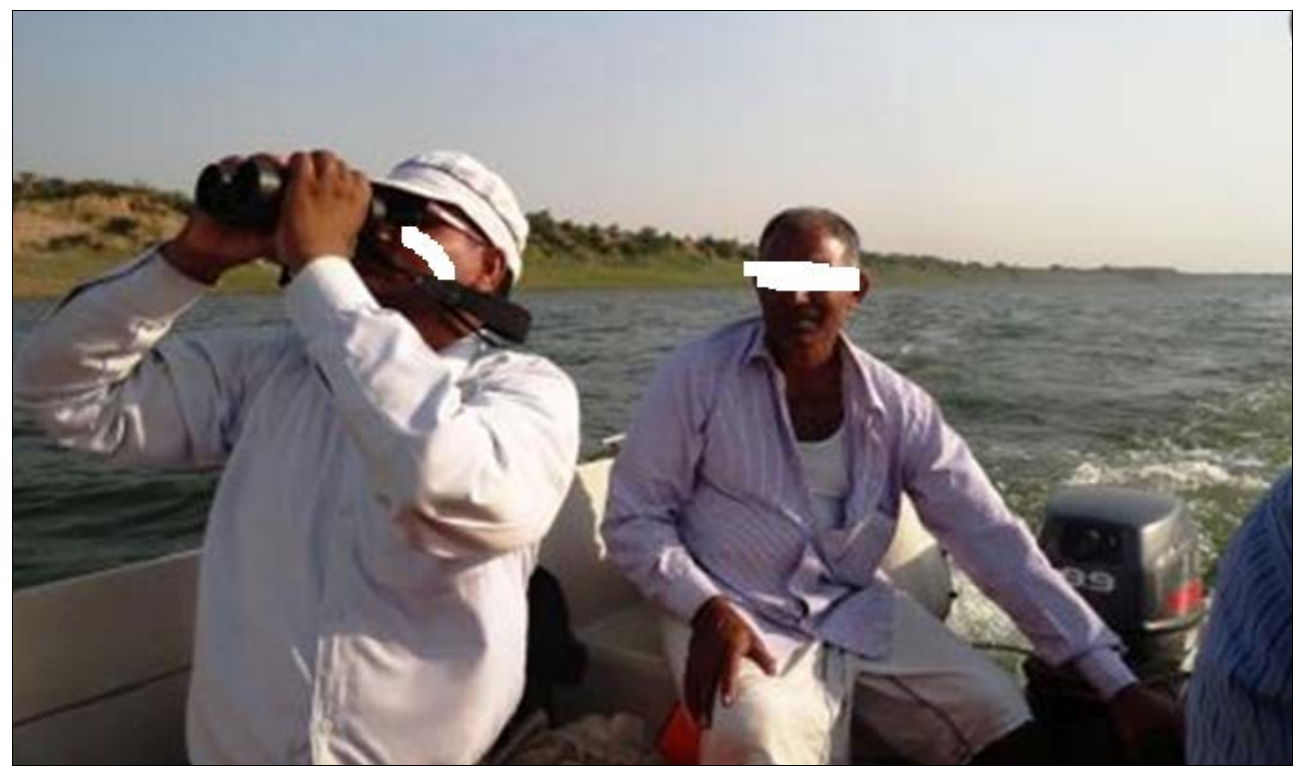

Fig 4: Chambal survey for Bird Exploration by C P Singh with collaboration of Etawah UPFD 
Table 1: List of Avian fauna their abundance, Categories habitat and Distribution

\begin{tabular}{|c|c|c|c|c|c|c|c|c|c|}
\hline S.No & Local name & Zoological name & Order & Family & Habitat & Category & \begin{tabular}{|c|} 
Observed \\
no
\end{tabular} & \begin{tabular}{|c|}
$\begin{array}{c}\text { IUCN Red } \\
\text { list category }\end{array}$ \\
\end{tabular} & Abundance \\
\hline 1 & Indian peafowl & Pavo cristatus & \multirow{5}{*}{ Galliformes } & \multirow{5}{*}{ Phasianidae } & $\mathrm{T}$ & $\mathrm{R}$ & 200 & \begin{tabular}{|c|} 
LC \\
\end{tabular} & Com \\
\hline 2 & Jungle Bush Quail & Perdicula asiatica & & & $\mathrm{T}$ & $\mathrm{R}$ & 300 & LC & com \\
\hline 3 & Grey francolin & $\begin{array}{c}\text { Francolinus } \\
\text { pondicerianus }\end{array}$ & & & $\mathrm{T} / \mathrm{F}$ & $\mathrm{R}$ & 10000 & LC & \\
\hline 4 & Common quail & Coturnix coturnix & & & $\mathrm{T} / \mathrm{F}$ & $\mathrm{R}$ & 15000 & $\mathrm{LC}$ & Com \\
\hline 5 & Rock bush quail & Perdicula argoondah & & & $\mathrm{T} / \mathrm{F}$ & $\mathrm{R}$ & 300 & LC & Com \\
\hline 6 & Rock pigeon & Columba livia & \multirow{5}{*}{ Collumbiforms } & \multirow{5}{*}{ Columbidae } & $\mathrm{T} / \mathrm{F}$ & $\mathrm{R}$ & 550 & $\mathrm{LC}$ & Un com \\
\hline 7 & Spotted dove & Spilopelia chinensis & & & $\mathrm{T}$ & $\mathrm{R}$ & 200 & LC & Un com \\
\hline 8 & Laughing dove & Spilopelia senegalensis & & & $\mathrm{T}$ & $\mathrm{R}$ & 450 & LC & Com \\
\hline 9 & $\begin{array}{c}\text { Eurasian collared } \\
\text { dove }\end{array}$ & Streptopelia decaocto & & & $\mathrm{T}$ & $\mathrm{R}$ & 300 & $\mathrm{LC}$ & Com \\
\hline 10 & $\begin{array}{c}\text { Yellow footed green } \\
\text { pigeon }\end{array}$ & Treron phoenicopterus & & & $\mathrm{T} / \mathrm{AE}$ & $\mathrm{R}$ & 100 & $\mathrm{LC}$ & Un com \\
\hline 11 & Green bee eater & Merops orientalis & \multirow{2}{*}{ Coraciiformes } & \multirow{2}{*}{ Meropidae } & $\mathrm{T} / \mathrm{F}$ & $\mathrm{R}$ & 600 & $\mathrm{LC}$ & Com \\
\hline 12 & Blue tailed bee eater & Merops philippinus & & & $\mathrm{T} / \mathrm{F}$ & $\mathrm{R}$ & 500 & LC & Com \\
\hline 13 & Indian roller & Coracias benghalensis & & Coracidae & $\mathrm{T}$ & $\mathrm{R}$ & 40 & LC & Un com \\
\hline 14 & Indian cuckoo & Eudynamy scolopaceus & \multirow{4}{*}{ Cuculiformes } & \multirow{4}{*}{ Cuculidae } & $\mathrm{T} / \mathrm{AE}$ & SV & 300 & LC & Com \\
\hline 15 & Drongo cuckoo & Surniculus lugubris & & & $\mathrm{T} / \mathrm{AE}$ & SV & 20 & LC & Un com \\
\hline 16 & Greater coucal & Centropus sinensis & & & $\mathrm{T} / \mathrm{F}$ & $\mathrm{R}$ & 150 & LC & Com \\
\hline 17 & Lesser coucal & Centropus bengalensis & & & $\mathrm{T} / \mathrm{F}$ & $\mathrm{R}$ & 203 & LC & Com \\
\hline 18 & Osprey & Pandion haliaetus & \multirow{9}{*}{ Falconiformes } & Pandionidae & T/RI & $\mathrm{R}$ & 10 & $\mathrm{LC}$ & Un com \\
\hline 19 & $\begin{array}{l}\text { Black shouldered } \\
\text { kite }\end{array}$ & Elanus axillaris & & \multirow{8}{*}{ Accipitridae } & $\mathrm{T}$ & $\mathrm{R}$ & 23 & $\mathrm{LC}$ & Un com \\
\hline 20 & Black kite & Milvus migrans & & & $\mathrm{T}$ & $\mathrm{R}$ & 40 & $\mathrm{LC}$ & Un com \\
\hline 21 & $\begin{array}{c}\text { Grey headed fish } \\
\text { eagle }\end{array}$ & $\begin{array}{l}\text { Ichthyophaga } \\
\text { ichthyaetus }\end{array}$ & & & T/RI & $\mathrm{R}$ & 15 & NT & Un com \\
\hline 22 & Egyptian vulture & Neophron percnopterus & & & T/RI & $\mathrm{R}$ & 400 & EN & Com \\
\hline 23 & Black eagle & Ictinaetus malaiensis & & & $\mathrm{T} / \mathrm{RI}$ & $\mathrm{R}$ & 63 & VU & Un com \\
\hline 24 & Shikra & Accipiter badius & & & $\mathrm{T}$ & & 201 & LC & com \\
\hline 25 & Besra & A.virgatus & & & $\mathrm{T}$ & $\mathrm{R}$ & 32 & $\mathrm{LC}$ & Un com \\
\hline 26 & $\begin{array}{l}\text { Long legged } \\
\text { buzzared }\end{array}$ & Buteo rufinus & & & $\mathrm{T}$ & $\mathrm{R} / \mathrm{LM}$ & 23 & $\mathrm{~T}$ & Un com \\
\hline 27 & Little egret & Egretta garzetta & \multirow{11}{*}{ Pelecaniformes } & \multirow{8}{*}{ Ardeidae } & WL & $\mathrm{R} / \mathrm{LM}$ & 2000 & $\mathrm{LC}$ & Com \\
\hline 28 & Median egret & Ardea intermedia & & & WL & $\mathrm{R} / \mathrm{LM}$ & 300 & LC & Com \\
\hline 29 & Large egret & Ardea alba & & & WL & $\mathrm{R} / \mathrm{LM}$ & 1500 & LC & Com \\
\hline 30 & Cattle egret & Bubulcus ibis & & & WL & $\mathrm{R} / \mathrm{LM}$ & 2500 & LC & Com \\
\hline 31 & Indian pond heron & Ardeola grayii & & & WL & $\mathrm{R} / \mathrm{LM}$ & 700 & LC & Com \\
\hline 32 & Night heron & Nycticorax nycticorax & & & WL & $\mathrm{R} / \mathrm{LM}$ & 35 & $\mathrm{LC}$ & Un com \\
\hline 33 & Grey heron & Ardea cinerea & & & WL & $\mathrm{R} / \mathrm{LM}$ & 300 & LC & Com \\
\hline 34 & Purple heron & Ardea pupurea & & & WL & $\mathrm{R} / \mathrm{LM}$ & 45 & LC & Un com \\
\hline 35 & Great white pelican & Pelecanus onocrotalus & & Pelecanidae & WL & $\mathrm{M}$ & 10 & $\mathrm{LC}$ & $\mathrm{R}$ \\
\hline 36 & Black ibis & Pseudibis papillosa & & \multirow[b]{2}{*}{ Threskionithidae } & WL & $\mathrm{R} / \mathrm{LM}$ & 800 & NT & Com \\
\hline 37 & Black headed ibis & $\begin{array}{c}\text { Threskiornis } \\
\text { melanocephalus }\end{array}$ & & & $\mathrm{WL}$ & $\mathrm{R} / \mathrm{LM}$ & 650 & NT & Com \\
\hline 38 & White necked stork & Ciconia episcopus & \multirow{7}{*}{ Ciconiiformes } & \multirow{4}{*}{ Ciconiidae } & WL & $\mathrm{R} / \mathrm{LM}$ & 1600 & VU & Com \\
\hline 39 & Black necked stork & $\begin{array}{l}\text { Ephippoiorhynchus } \\
\text { asiaticus }\end{array}$ & & & WL & $\mathrm{R} / \mathrm{LM}$ & 8 & $\mathrm{~T}$ & $\mathrm{R}$ \\
\hline 40 & Painted stork & Mycteria leucocephala & & & WL & $\mathrm{R} / \mathrm{LM}$ & 1900 & $\mathrm{~T}$ & Com \\
\hline 41 & Asian Open bill stork & Anastomus oscitans & & & WL & $\mathrm{R} / \mathrm{LM}$ & 200 & LC & Com \\
\hline 42 & Common king fisher & Alcedo atthis & & \multirow{3}{*}{ Alcedinidae } & WL & $\mathrm{R}$ & 230 & $\mathrm{LC}$ & Com \\
\hline 43 & $\begin{array}{c}\text { White throated king } \\
\text { fisher }\end{array}$ & Halcyon smyrnensis & & & $\mathrm{WL}$ & $\mathrm{R} / \mathrm{LM}$ & 400 & NT & Com \\
\hline 44 & Pied kingfisher & Ceryie rudis & & & WL & $\mathrm{R} / \mathrm{LM}$ & 50 & $\mathrm{LC}$ & Un com \\
\hline 45 & Little cormorant & Microcarbo niger & Suliformes & & WL & $\mathrm{R} / \mathrm{LM}$ & 2400 & LC & Com \\
\hline 46 & Great cormorant & Phlacrocorax carbo & & Phalacrocorcidae & WL & $\mathrm{R} / \mathrm{LM}$ & 300 & LC & Un com \\
\hline 47 & Darter & Anhinga anhinga & & Anhingidae & WL & $\mathrm{R} / \mathrm{LM}$ & 145 & NT & Com \\
\hline 48 & Sarus crane & Grus antigone & & Gruidae & WL & $\mathrm{R} / \mathrm{LM}$ & 400 & VU & Com \\
\hline 49 & Domicile crane & Grus virgo & & Gruidae & WL & $\mathrm{R} / \mathrm{LM}$ & 1 & $\mathrm{LC}$ & $\mathrm{R}$ \\
\hline 50 & $\begin{array}{c}\text { White breasted water } \\
\text { hen }\end{array}$ & $\begin{array}{l}\text { Amaurornis } \\
\text { phoenicurus }\end{array}$ & Gruiformes & Ralide & $\mathrm{WL}$ & $\mathrm{R}$ & 200 & LC & Com \\
\hline 51 & Purple Moorhen & Porphyrio porphyrio & & & WL & $\mathrm{R}$ & 170 & LC & Com \\
\hline 52 & Black winged stilt & Himantopus himantopus & & Recurvirostridae & WL & $\mathrm{R}$ & 375 & LC & Com \\
\hline 53 & Eurasian thick knee & Burhinus oedicnemus & & \begin{tabular}{|l|} 
Burhinidae \\
\end{tabular} & $\mathrm{T}$ & $\mathrm{R}$ & 35 & LC & Un com \\
\hline 54 & River lapwing & Venellus duvaucelii & adriliforn & & WL & $\mathrm{R}$ & 200 & $\mathrm{~T}$ & Com \\
\hline 55 & Red wetted lapwing & Vanellus indicus & & adriidae & $\mathrm{T}$ & $\mathrm{R}$ & 245 & LC & Com \\
\hline
\end{tabular}




\begin{tabular}{|c|c|c|c|c|c|c|c|c|c|}
\hline 56 & $\begin{array}{l}\text { Yellow wetted } \\
\text { lapwing }\end{array}$ & Vanellus malabaricus & & & $\mathrm{T}$ & $\mathrm{R}$ & 30 & $\mathrm{LC}$ & Un com \\
\hline 57 & Common sand piper & Actitus hypoleucos & & \multirow{4}{*}{ Scolopacidae } & WL & $\mathrm{R} / \mathrm{LM}$ & 211 & $\mathrm{LC}$ & Com \\
\hline 58 & Common snipe & Gallinago gallinago & & & WL & $\mathrm{R} / \mathrm{LM}$ & 303 & $\mathrm{~T}$ & Com \\
\hline 59 & Grey plover & Pluvialis squatarola & & & WL & $\mathrm{R} / \mathrm{LM}$ & 186 & $\mathrm{LC}$ & Com \\
\hline 60 & Yellow legged gull & Larus mechahellis & & & WL & $\mathrm{R} / \mathrm{LM}$ & & $\mathrm{T}$ & Un com \\
\hline 61 & River turn & Sterna aurantia & & \multirow{2}{*}{ Laridae } & WL & $\mathrm{R}$ & 54 & VU & Un com \\
\hline 62 & Indian skeemer & Rynchops albicollis & & & WL & $\mathrm{R}$ & 5 & VU & $\mathrm{R}$ \\
\hline 63 & House swift & Apus nipalensis & Apodiformes & Apodidae & $\mathrm{AE}$ & $\mathrm{R}$ & 255 & $\mathrm{LC}$ & Com \\
\hline 64 & Jungle babbler & Turdoides striata & \multirow{17}{*}{ Passeriformes } & Leiothrichidae & $\mathrm{T} / \mathrm{F}$ & $\mathrm{R}$ & 400 & $\mathrm{LC}$ & Com \\
\hline 65 & Large grey babbler & T.malcolmi & & & $\mathrm{T} / \mathrm{F}$ & $\mathrm{R}$ & 200 & $\mathrm{LC}$ & Com \\
\hline 66 & Tailor bird & Orthotomus sutorius & & Cisticolidae & $\mathrm{T} / \mathrm{F}$ & S V & 2 & $\mathrm{LC}$ & $\mathrm{R}$ \\
\hline 67 & Weaver bird & Ploceus phillippinus & & Ploceidae & $\mathrm{T} / \mathrm{F}$ & SV & & $\mathrm{LC}$ & \\
\hline 68 & House crow & Corvus splendens & & \multirow{2}{*}{ Corvidae } & $\mathrm{T}$ & $\mathrm{R}$ & 2300 & LC & Com \\
\hline 69 & Rufous treepie & Dendrocitta vagabunda & & & $\mathrm{T}$ & $\mathrm{R} / \mathrm{LM}$ & 39 & $\mathrm{LC}$ & Un com \\
\hline 70 & Jungle crow & Corvus macrorhynchos & & \multirow{4}{*}{ Sturnidae } & $\mathrm{T}$ & $\mathrm{R}$ & 57 & $\mathrm{LC}$ & Un com \\
\hline 71 & Pied myna & Gracupica contra & & & $\mathrm{T}$ & $\mathrm{R}$ & 200 & LC & Com \\
\hline 72 & Jungle myna & Acridotheres fuscus & & & $\mathrm{T}$ & $\mathrm{R}$ & 234 & LC & Com \\
\hline 73 & Common myna & A.tristis & & & $\mathrm{T}$ & $\mathrm{R}$ & 567 & $\mathrm{LC}$ & Com \\
\hline 74 & Oriental white eye & Zosterosps palpebrosus & & Zosteropidae & $\mathrm{T}$ & $\mathrm{R}$ & 50 & Lc & Com \\
\hline 75 & Black Drongo & Dicrurus macrocercus & & Dicruridae & $\mathrm{T}$ & $\mathrm{R}$ & 333 & LC & Com \\
\hline 76 & $\begin{array}{c}\text { Greater Racket tailed } \\
\text { Drongo }\end{array}$ & D.paradiseus & & & $\mathrm{T}$ & $M$ & 3 & $\mathrm{LC}$ & $\mathrm{R}$ \\
\hline 77 & $\begin{array}{c}\text { Oriental Magpie } \\
\text { Robin }\end{array}$ & Copsychus saularis & & Muscicapidae & $\mathrm{T} / \mathrm{F}$ & $\mathrm{R}$ & 322 & LC & Com \\
\hline 78 & Red vented Bulbul & Pycnonotus cafer & & Pycnonotidae & $\mathrm{T} / \mathrm{F}$ & $\mathrm{R}$ & 167 & $\mathrm{LC}$ & Com \\
\hline 79 & Forest wagtail & Dandronanthus indicus & & Motacillidae & $\mathrm{T} / \mathrm{F}$ & $\mathrm{R}$ & 344 & $\mathrm{LC}$ & Com \\
\hline 80 & House sparrow & Passer domesticus & & Passeridae & $\mathrm{T}$ & $\mathrm{R}$ & 432 & $\mathrm{E}$ & Com \\
\hline 81 & Eurasian Eagle owl & Bubo bubo & \multirow{2}{*}{ Strigiformes } & \multirow{2}{*}{ Strigidae } & $\mathrm{T}$ & $\mathrm{R} / \mathrm{LM}$ & 43 & $\mathrm{LC}$ & Un com \\
\hline 82 & Motteled Wood Owl & Strix ocellata & & & $\mathrm{T} / \mathrm{F}$ & $\mathrm{R}$ & 34 & $\mathrm{~T}$ & Un com \\
\hline 83 & Common Hoopoe & Upupa epops & Bucerotiformes & Upupidae & $\mathrm{T} / \mathrm{F}$ & $\mathrm{R}$ & 143 & $\mathrm{LC}$ & Com \\
\hline 84 & Indian Grey Hornbill & Ocyceros birostris & & Bucerotidae & $\mathrm{T}$ & LM & 232 & $\mathrm{LC}$ & Com \\
\hline 85 & Rose ringed parakeet & Psittacula krameri & Psittaciformes & Psittaculidae & $\mathrm{T} / \mathrm{F}$ & $\mathrm{R}$ & 1233 & $\mathrm{LC}$ & Com \\
\hline
\end{tabular}

Habitat: $\mathrm{WL}=$ Wet land, $\mathrm{RI}=$ Riverine, $\mathrm{T}=$ Terrestrial, $\mathrm{F}=$ forest, $\mathrm{AE}=$ Aerial

Category: $\mathrm{R}=$ Resident, $\mathrm{LM}=$ Local migrant, $\mathrm{SM}=$ summer visitor, $\mathrm{M}=$ Migrant and $\mathrm{WM}=$ winter visitor

IUCN Status L: LC = Least concern, VU = Vulnerable, EN = Endangered, NT =near threatened, T= Threatened

Abundance: Com $=$ Common, Un com $=$ Uncommon, $\mathrm{R}=$ Rare

Table: 2 No of families and genus under various orders

\begin{tabular}{|c|c|c|c|c|c|}
\hline S. No. & Order & No of Family & No of Genus & No of Species & Total \\
\hline 1 & Galliformes & 1 & 4 & 5 & 5 \\
\hline 2 & Collumbiformes & 1 & 4 & 5 & 5 \\
\hline 3 & Coraciformes & 2 & 2 & 3 & 3 \\
\hline 4 & Cuculiformes & 1 & 3 & 4 & 4 \\
\hline 5 & Falconiformes & 2 & 8 & 9 & 9 \\
\hline 6 & Pelecaniformes & 3 & 8 & 11 & 11 \\
\hline 7 & Ciconiformes & 2 & 7 & 7 & 7 \\
\hline 8 & Suliformes & 2 & 3 & 3 & 3 \\
\hline 9 & Gruiformes & 2 & 4 & 4 & 4 \\
\hline 10 & Charadriiformes & 5 & 9 & 11 & 11 \\
\hline 11 & Apodiformes & 1 & 1 & 1 & 1 \\
\hline 12 & Passeriformes & 11 & 14 & 17 & 17 \\
\hline 13 & Strigiformes & 1 & 2 & 2 & 2 \\
\hline 14 & Bucerotiformes & 2 & 2 & 2 & 2 \\
\hline 15 & Psittaciformes & 1 & 1 & 1 & 1 \\
\hline Total & 15 & 37 & 72 & 85 & 85 \\
\hline
\end{tabular}

Table 3: Number and percentage of different species of various orders

\begin{tabular}{|c|c|c|c|c|c|}
\hline S. No & Order & No of families & \% 0f families & No of genus & \% of genus \\
\hline 1 & Galliformes & 1 & 2.70 & 4 & 5.55 \\
\hline 2 & Gruiformes & 1 & 2.70 & 4 & 2.55 \\
\hline 3 & Collumbiforms & 2 & 5.40 & 3 & 2.77 \\
\hline 4 & Ciconiiformes & 1 & 2.70 & 8 & 4.16 \\
\hline 5 & Coraciiformes & 2 & 5.40 & 8 & 11.11 \\
\hline 6 & Cuculiformes & 3 & 8.10 & 7 & 11.11 \\
\hline 7 & Falconiformes & 2 & 5.40 & 3 & 9.72 \\
\hline 8 & Pelecaniformes & 2 & 5.40 & & 4.16 \\
\hline
\end{tabular}




\begin{tabular}{|c|c|c|c|c|c|}
\hline 9 & Suliformes & 2 & 5.40 & 4 & 5.55 \\
\hline 10 & Charadriiformes & 5 & 13.51 & 9 & 12.5 \\
\hline 11 & Apodiformes & 1 & 2.70 & 14 & 1.38 \\
\hline 12 & Passeriformes & 11 & 29.72 & 2 & 19.44 \\
\hline 13 & Strigiformes & 1 & 2.70 & 2 & 2.77 \\
\hline 14 & Bucerotiformes & 2 & 5.40 & 1 & 2.77 \\
\hline 15 & Psittaciformes & 1 & 2.70 & & 1.38 \\
\hline
\end{tabular}

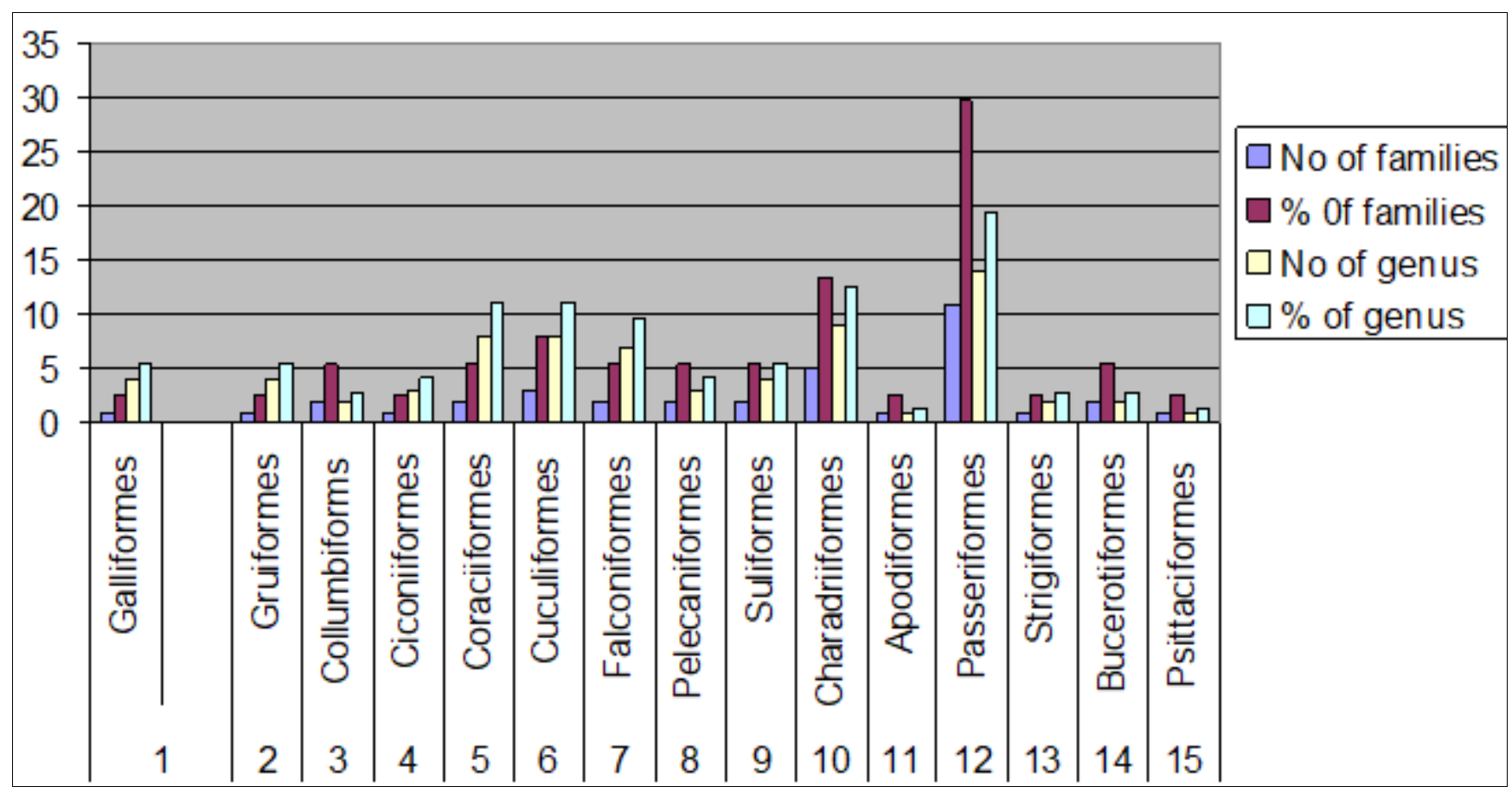

Fig 5: Figure shows in Galliformes, Gruiformes etc.

Table 4: No of species and percentage of various families under different orders

\begin{tabular}{|c|c|c|c|c|c|c|c|}
\hline S.No & Family & No of species & $\%$ of family & S.No. & Family & No of species & $\%$ of family \\
\hline 1 & Phasianidae & 5 & 5.88 & & & & \\
\hline 2 & Columbidae & 5 & 5.88 & 20 & Scolopacidae & 4 & 4.70 \\
\hline 3 & Meropidae & 2 & 2.35 & 21 & Laridae & 2 & 2.35 \\
\hline 4 & coracidae & 1 & 1.17 & 22 & Apodidae & 1 & 1.17 \\
\hline 5 & Cuculidae & 4 & 4.70 & 23 & Leiothrichidae & 2 & 2.35 \\
\hline 6 & Pandionidae & 1 & 1.17 & 24 & Cisticolidae & 1 & 1.17 \\
\hline 7 & Accipitridae & 8 & 9.41 & 25 & Ploceidae & 1 & 1.17 \\
\hline 8 & Ardeidae & 8 & 9.41 & 26 & Corvidae & 2 & 2.35 \\
\hline 9 & Pelecanidae & 1 & 1.17 & 27 & Sturnidae & 4 & 4.70 \\
\hline 10 & Threskionithidae & 2 & 2.35 & 28 & Zosteropidae & 1 & 1.17 \\
\hline 11 & Ciconiidae & 4 & 4.70 & 29 & Dicruridae & 2 & 2.35 \\
\hline 12 & Alcedinidae & 3 & 3.52 & 30 & Muscicapidae & 1 & 1.17 \\
\hline 13 & Phalacrocorcidae & 2 & 2.35 & 31 & Pycnonotidae & 1 & 1.17 \\
\hline 14 & Anhingidae & 1 & 1.17 & 32 & Motacillidae & 1 & 1.17 \\
\hline 15 & Gruidae & 2 & 2.35 & 33 & Passeridae & 1 & 1.17 \\
\hline 16 & Ralide & 2 & 2.35 & 34 & Strigidae & 2 & 2.35 \\
\hline 17 & Recurvirostridae & 1 & 1.17 & 35 & Upupidae & 1 & 1.17 \\
\hline 18 & Burhinidae & 1 & 1.17 & 36 & Bucerotidae & 1 & 1.17 \\
\hline 19 & Charadriidae & 3 & 3.52 & 37 & Psittaculidae & 1 & 1.17 \\
\hline
\end{tabular}

Table 5: Habitat and threats

\begin{tabular}{|c|c|c|}
\hline S.No. & Type of habitat & Observed threats \\
\hline 1 & Terrestrial & Deforestation, Poaching, electric wires, use of fertilizers and other chemicals in crops anthropogenic activities \\
\hline 2 & Aerial & Deforestation, electric wire and telephone tower \\
\hline 3 & Forest & Deforestation, mining poaching, fires \\
\hline 4 & Wet land & $\begin{array}{c}\text { Deforestation, Water lifting, poaching, agricultural practices use of chemicals in crop fields, fishing, polythene in } \\
\text { water bodies and sewage electric wires }\end{array}$ \\
\hline 5 & River & Deforestation, Fishing, sand mining, poaching, Sewage, polythene, chemicals \\
\hline 6 & Ponds & Deforestation, Mining, water lifting, fishing, use chemicals, polythene, chemicals sewage \\
\hline
\end{tabular}




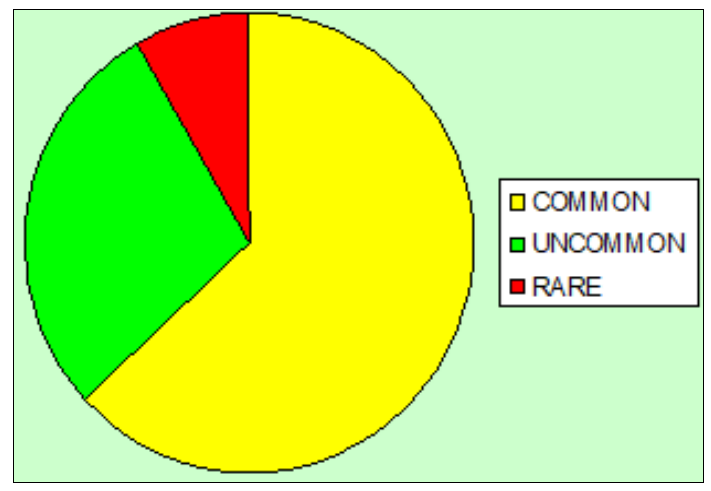

Abundance Fig 6: common $=62.52 \%$, un common $28.25 \%$, rare $8.25 \%$

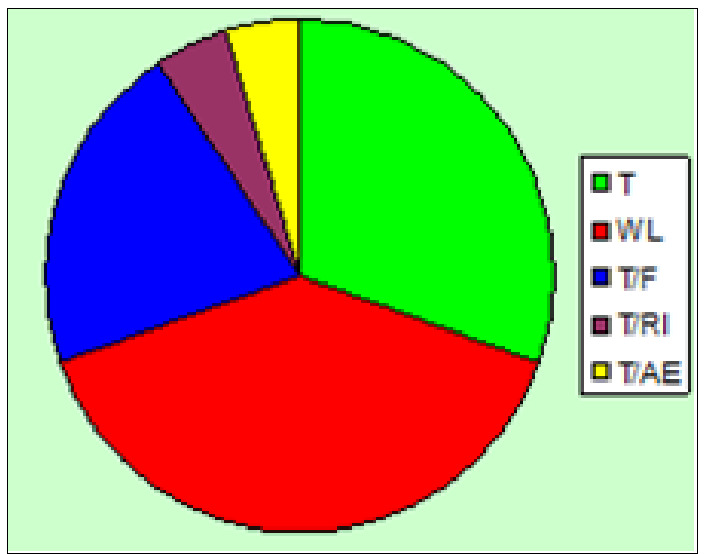

Habitat Fig 7: $\mathrm{T}=30.58 \%, \mathrm{WL}=38.82 \%, \mathrm{~T} / \mathrm{RI}=4.70 \%, \mathrm{~T} / \mathrm{F}=21.17 \%$, $\mathrm{T} / \mathrm{AE}=4.70 \%$

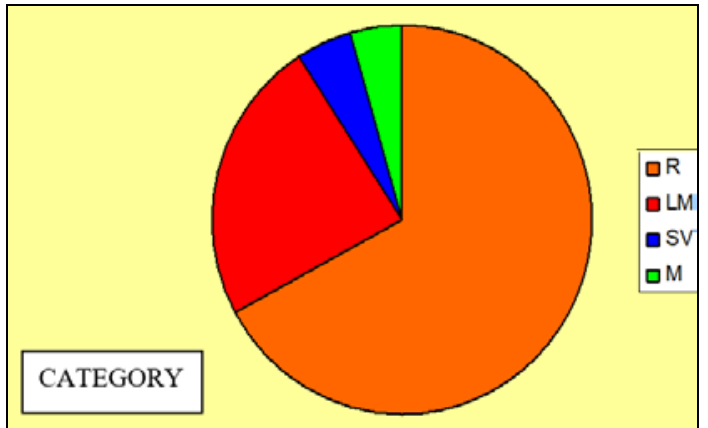

Fig 8: $\mathrm{R}=69.41 \%, \mathrm{LM}=24.70 \%, \mathrm{SV}=4.70 \%, \mathrm{M}=1.17 \%, \mathrm{WV}=0 \%$

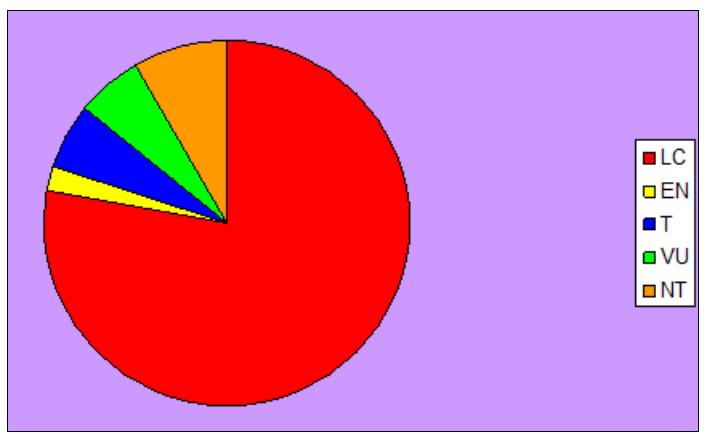

Fig 9: $\mathrm{LC}=77.64 \%, \mathrm{EN}=2.43 \% \mathrm{VU}=5.88 \%, \mathrm{NT}=5.88 \%, \mathrm{~T}=8.23 \%$

\section{Discussion}

The similar study was various researchers, a total 97 bird species were recorded and belonging to 78 genera, 41 families and over 20 orders were noticed. Of these 97 bird species in Dabarusinghi reservoir ${ }^{[1]}$ \{The stautus and distribution of chambal river was studied by C.P Singh et al. ${ }^{[2]}$ where as 124 bird species belonging to the 52 families were recorded across Anantapuramu dt. Accipitridaewas found to be the most dominant family consisting of 16 species in the study area ${ }^{[3]}$. Rubina and Ganesh studied on Avifaunal diversity status in lakes of Dharwad, Karnataka State ${ }^{[4]}$. The water bodies supports a rich biodiversity with a

The study revealed presence of 311 species, belonging to 18 Orders and 70 families in different habitats in and around KVNP and MFR. Out of these 311 species, 10 species ${ }^{[5]}$ Wetlands directly and indirectly support of birds by providing ecological services ${ }^{[6]}$. Presently human and manmadeanthropogenic activities cause alterations of wetlands. Changes in wetland. Arish et al. studied wetland ecosystem. During the study of shivaji prabhaker chaavan study 105 species belongs to 14 Orders and 40 Families were recorded in winter season, year 2018, out of that the distribution and characteristics was 64 (60.9\%) migratory, 41 (39.0\%) Resident, 04 (3.8\%) Near Threatened, 6 (5.17\%) Abundant, 36 (34.2\%) Occsional, 43 (40.9\%) Common, 20 (19.0\%) Rare. Winter migratory species were 16 (15.2\%). Maximum number was of different duck species (Ansariformes) with species diversity 14 (14.3\%). Winter season is population and diversity rich period fish and algae form major food for birds. black necked stork thet is $\mathrm{Nt}$ species incounter thrice in survey domicile crane observe only once in chambal survey taylor bird ony sighted once in forest area.in frest of chambal nregion found many hanging nest of weaver birds.

The highest species diversity was observed during the wet period. months of November December and also in Monsoon period The large group of wetland birds consists mainly of cormorants Darters opinbills painted storks, sarus crane, moorhens eagrets, black necked storks, lapwings pond herons, water hens black and white ibis sand pipper kingfishers etc whlile in terrestrial habitats main birds were webler, Indian peafowl, Coucal, Paroot pigeon doves hope pheasants hoopoe mynas etc. The population of anadidae is highest in migrant.

\section{Conclusion}

New small water bodies of kaswa Bakewar, Ajitmal Ekdil, road and canal side Chambal and Yamuna, Sanger river plays a vital role in the annual cycle of the non-migratory birds and serves breeding, feeding, moving, mating grounds for several resident species. Birds either resident or migratory according to their occurrence, frequency and population they have been assigned various categories. Order Passeriformes was more dominant in the total population. Status of birds was classified as Resident species, Local migratory (Resident of India) and winter and Summer visitors (International) which are found in the study area only certain period. River Chambal is good abode for many species but specially birds. Indian national bird peacock were found to be searching new habitat around human habitat due to disturbance of natural habitat due to deforestation. Population of passer domesticus is higher in forest than villages.

\section{Acknowledgement}

The authors team is highly thankful to the Principal and general secretary of Janta Maha Vidyalaya Ajitmal Auraiya for permitting to us for this study. We are also highly grateful to Mr. S.B, Mishra (retired depuity forest ranger from NCS) for their rigorous support for field survey. The authors would like to thanks my colleague Sachin Jain who support me for 
this study.

\section{References}

1. Grimmett R, Inskipp C, Inskipp T. Birds of the Indian Subcontinent. Oxford University Press, New Delhi. 1998.

2. Ali S, 1996. The Book of Indian Birds, $13^{\text {th }}$ edition, Bombay Natural History Society \& Oxford University Press, Bombay. 1941, 1-412.

3. Al S, Ripley SD. The books of the bird of India and Pakistan Oxford University Press. Bombay, London, New York. 1968; 74:1-10.

4. Peterson AW. Destruction of birdlife in Minnesota IV. Birds killed at Park Rapids. Flicker. 1963;35(4):113.

5. Rahmani Asad R, Gayatri Ugra. Birds of wetlands and Grasslands. 1980, 43.

6. Ehrlich PR, Dobkin DS, Wheye D, Pimm SL. The Bird Watcherees Handbook: A Guide to the Natural History of the Birds of Britain and Europe" Oxford University Press, Oxford, 1994.

7. Kazmierczak K, Singh RA. Bird Watchers Guide to India UK. Ali, Ripley. Hand Book of the Birds of India and Pakistan. 2001;6:77.

8. Kazmierczak K, Van Perto B. Field Guide to the Birds ofthe Indian Subcontinent, 2000.

9. Ali S. The Book of Indian Birds, 13thedition, Bombay Natural History Society \& Oxford University Press, Bombay. 1941-1996, 1-412

10. Grimmet RC, Inskipp Inskipp T. Birds of Indian Subcontinent Oxford University Press. Delhi. 2001, 384.

11. Manakkadan R, Pittie A. Standardized common and scientific names of the birds of the Indian subcontinent” Buceros. 2001;6(1):1-37.

12. Inskipp $\mathrm{T}$, Lindsey $\mathrm{N}$, Duckworth XW. An annotated checklist of the birds of the oriental region Oriental Birds Club, Sandy U.K., 1994.

13. Bala Subramanyam VV. Avifaunal diversity of Anantapuramu (semi-arid) District, Andhra Pradesh India. International Journal of Zoology and Applied Biosciences. 2018; 3(2):426-439.

14. Rubina M, Nadaf CB, Ganesh D. A Study on Avifaunal Diversity Status in Lakes of Dharwad, Karnataka State. J Ecophysiol. Occup. Hlth. 2016;16(1, 2):13-21.

15. Krishnan M. Birds of Southern India, 1981;CII23:26-31.

16. Sbbarao G, Bhaskar MK. Birds and Bird Watching In India $1^{\text {ST }}$ edition 2019 graphic media Bengaluru Karnataka. 